\title{
ANNEALED ALLOY OF ISING MAGNETS WITHIN TWO-SITE CLUSTER APPROXIMATION
}

\author{
R.R. LEvitskil, S.I. Sorokov aNd R.O. SoKolovskiI \\ Institute for Condensed Matter Plysics of the National Academy of Sciences \\ 1 Svientsitski str., Lviv 290011, Ukraine
}

Ising mixture with equilibrium disorder is considered. The nearest neighbour spin coupling and direct interatomic interaction are taken into account within two-site cluster approximation. Thermodynamic potential and $q$-dependent pair correlation function are obtained. Interplay between interspin and interatomic interactions, their influence on magnetic phase transition and alloy segregation are studied. Cluster fluctuations cause significant changes with respect to mean field theory: phase diagram complicates and magnetic properties deviate from those of quenched system.

PACS numbers: $64.60 . \mathrm{Cn}$

We shall consider an Ising mixture with the Hamiltonian $H$

$$
H=-\sum_{i} \kappa_{i} S_{i}-\frac{1}{2} \sum_{i j} K_{i j} S_{i} S_{j}-\sum_{i} \mu_{i}-\frac{1}{2} \sum_{i j} V_{i j} .
$$

It describes a spin system on the sites of a simple lattice $(i, j=1 \ldots N)$ with ferromagnetic interaction $K_{i j}\left(K_{i j} \geq 0\right)$ in the site dependent field $\kappa_{i}$. The set of spin variables $\left\{S_{i}\right\}$ represents a state of the spin system $\left(S_{i}= \pm 1\right)$. The Hamiltonian includes also "nonexchange" pair interaction $V_{i j}$ and field $\mu_{i}$. Each site contains a spin of a certain sort and the sort configuration is described by the set of variables $\left\{X_{i \alpha}\right\}\left(\alpha=1 \ldots \Omega, \Omega\right.$ is a number of sorts): $X_{i \alpha}=1$ if the site $i$ is occupied by the spin of sort $\alpha$, otherwise $X_{i \alpha}=0$. Interactions $\kappa_{i}, K_{i j}, \mu_{i}, V_{i j}$ depend on the sort configuration

$$
\begin{aligned}
\kappa_{i} & =\sum_{\alpha} \kappa_{i \alpha} X_{i \alpha}, \quad K_{i j}=\sum_{\alpha \beta} K_{\alpha \beta} X_{i \alpha} X_{j \beta}, \quad \mu_{i}=\sum_{\alpha} \mu_{i \alpha} X_{i \alpha}, \\
V_{i j} & =\sum_{\alpha \beta} V_{\alpha \beta} X_{i \alpha} X_{j \beta} .
\end{aligned}
$$

Couplings $K_{i j}, V_{i j}$ are assumed to be nonzero only for the nearest neighbour sites. The model is usually considered in two limits. In the quenched limit the sort configuration is frozen. In the annealed case an equilibrium sort configuration is realized and the system is described by the density matrix 


$$
\rho(\{S, X\})=Z^{-1} \exp (-\beta H), \quad Z=\mathrm{p}_{\{S, X\}} \exp (-\beta H), \quad \beta=\frac{1}{k_{\mathrm{B}} T},
$$

as well as by the generating function

$$
F(\{i, \mu\})=\ln Z=-\beta \mathcal{G},
$$

where $\mathcal{G}$ is the thermodynamic potential of the system.

The generating function allows to calculate the correlation functions of the system

$$
\left\langle S_{i_{1} \alpha_{1}} \ldots S_{i_{n} \alpha_{n}} X_{j_{1} \beta_{1}} \ldots X_{j_{1} \beta_{1}}\right\rangle^{c}=\frac{1}{\beta^{n+l}} \frac{\delta}{\delta \kappa_{i_{1} \alpha_{1}}} \ldots \frac{\delta}{\delta \kappa_{i_{n} \alpha_{n}}} \frac{\delta}{\delta \mu_{j_{1} \beta_{1}}} \ldots \frac{\delta}{\delta \mu_{j_{1} \beta_{l}}} F,
$$

where $S_{i \alpha}=S_{i} X_{i \alpha}$, the superscript ${ }^{c}$ means cumulant averaging and

$$
\langle(\cdots)\rangle=\operatorname{Sp}_{\{S, X\}}[\rho(\{S, X\})(\cdots)] .
$$

The chemical potentials $\mu_{i \alpha}$ have to be found from the equations $\left\langle X_{i \alpha}\right\rangle=c_{\alpha}$, where $c_{\alpha}$ is a concentration of the sort $\alpha$ spins.

With the accuracy to the first term of cluster expansion [1] (two-site cluster) we obtain the generating function in the form

$$
F=(1-z) \sum_{i} F_{i}+\frac{1}{2} \sum_{i} \sum_{j \in \pi_{i}} F_{i j},
$$

where $\pi_{i}$ is a set of the nearest neighbours to the site $i, z$ is the first coordination number of the lattice and

$$
\begin{aligned}
& F_{i}=\ln Z_{i}, \quad Z_{i}=\operatorname{Sp}_{S_{i} X_{i}} \exp \left(-\beta I I_{i}\right), \quad H_{i}=-\bar{\kappa}_{i} S_{i}-\mu_{i}, \\
& F_{i j}=\ln Z_{i j}, \quad Z_{i j}=\operatorname{Sp}_{S_{i} X_{i} S_{j} X_{j}} \exp \left(-\beta H_{i j}\right), \\
& H_{i j}=-{ }_{i} \bar{\kappa}_{j} S_{i}-{ }_{j} \bar{\kappa}_{i} S_{j}-K_{i j} S_{i} S_{j}-{ }_{j} \bar{\mu}_{i}-\bar{\mu}_{i}-V_{i j}, \\
& \bar{\kappa}_{i}=\kappa_{i}+\sum_{r \in \pi_{i}}{ }_{r} \bar{\varphi}_{i}, \bar{\mu}_{i}=\mu_{i}+\sum_{r \in \pi_{i}}{ }_{r} \bar{\psi}_{i}, r \bar{\kappa}_{i}=\bar{\kappa}_{i}-{ }_{r} \bar{\varphi}_{i}, \quad r \bar{\mu}_{i}=\bar{\mu}_{i}-{ }_{r} \bar{\psi}_{i}, \\
& { }_{r} \bar{\varphi}_{i}=\sum_{\alpha} X_{i \alpha} \bar{\varphi}_{i \alpha}, \quad{ }_{r} \bar{\psi}_{i}=\sum_{\alpha} X_{i \alpha} \bar{\psi}_{i \alpha} .
\end{aligned}
$$

In the effective IIamiltonians we have incorporated the variational parameters $\bar{\varphi}, \bar{\psi}$ which should minimize the thermodynamic potential

$$
\frac{\partial F}{\partial_{r} \bar{\varphi}_{i \alpha}}=0, \quad \frac{\partial F}{\partial_{r} \bar{\psi}_{i \alpha}}=0 .
$$

These conditions yield the following self-consistency equations for the average values:

$$
\left\langle S_{i \alpha}\right\rangle=\left\langle S_{i \alpha}\right\rangle_{H_{i}}=\left\langle S_{i \alpha}\right\rangle_{H_{i j}}, \quad\left\langle X_{i \alpha}\right\rangle=\left\langle X_{i \alpha}\right\rangle_{H_{i}}=\left\langle X_{i \alpha}\right\rangle_{H_{i j}},
$$

where

$$
\langle\cdots\rangle_{H_{i}}=\operatorname{Sp} Z_{i}^{-1} \exp \left(-\beta H_{i}\right)(\cdots), \quad\langle\cdots\rangle_{H_{i j}}=\operatorname{Sp} Z_{i j}^{-1} \exp \left(-\beta H_{i j}\right)(\cdots),
$$

Equations (10) imply the equality of the one-site and intracluster distribution functions. In the one-site IIamiltonian contributions of all nearest neighbours are replaced with effective fields $\bar{\varphi}=\bar{\varphi}(\{\kappa, \mu\}), \bar{\psi}=\bar{\psi}(\{\kappa, \mu\})$; in the cluster Hamiltonian one bond is accounted explicitly. In the spatially uniform case 


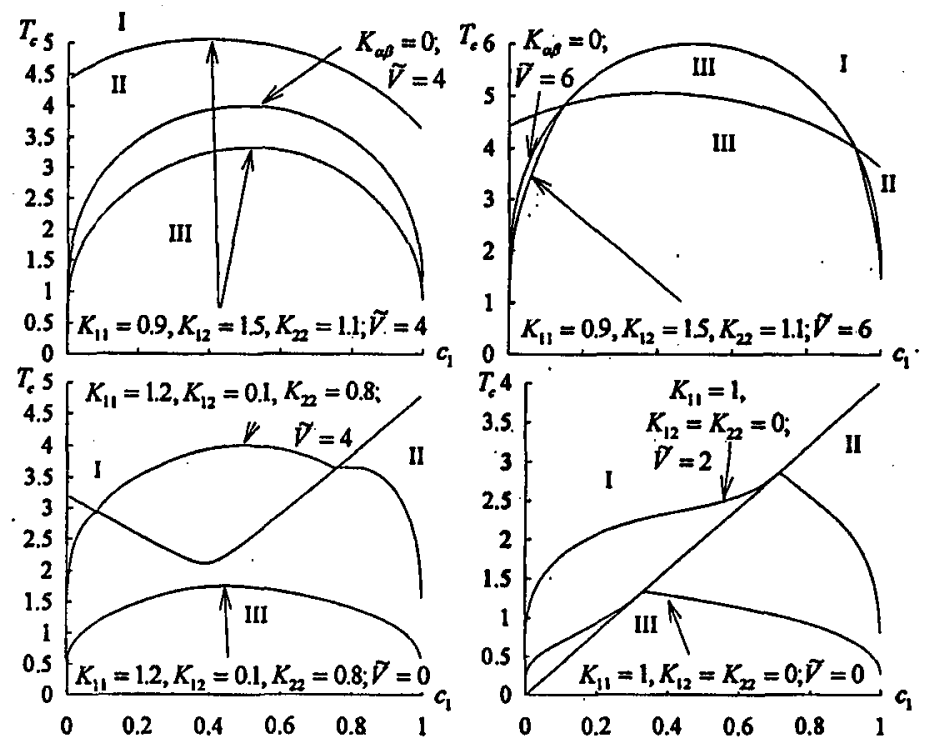

Fig. 1. Phase diagrams of the model on the plane square lattice $(z=4)$ within MFA [6] at different model parameters. I - paramagnetic phase, II - magnetized phase, III segregated pliase. The line of magnetic transitions (which separates regions I and II) is virtual in the segregated phase. MFA predicts that magnetic line is not affected by the "nonexchange" interactions $V_{\alpha \beta}$ and magnetism affects segregation only in magnetized phase. Both these rules are broken in TCA (Fig. 2).
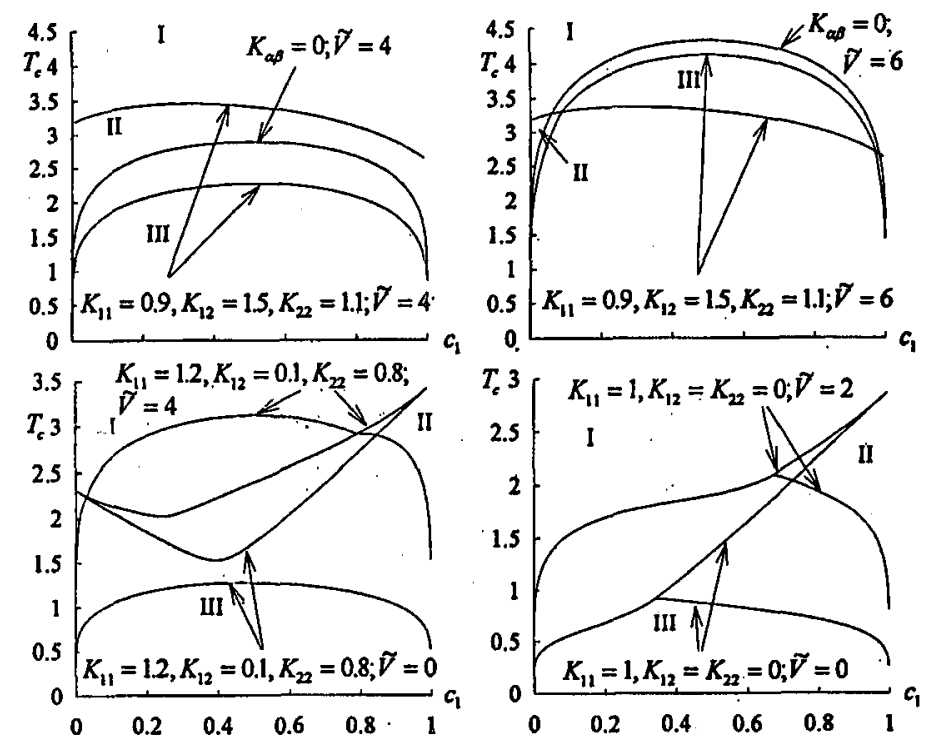

Fig. 2. Phase diagrams within TCA at different model parameters.

$\left(\kappa_{i \alpha}=\kappa_{\alpha}, \mu_{i \alpha}=\mu_{\alpha}, r \bar{\varphi}_{i \alpha}=\bar{\varphi}_{\alpha}, r_{r} \bar{\psi}_{i \alpha}=\bar{\psi}_{\alpha}\right)$ Eqs. (10) yield a well known result of the Bethe approximation [1]. The advantage of our approach consists in 
spatial nonuniformity of Eqs. (10). It allows us to calculate correlation functions of the model with differentiation of the generating function [2,3]. For example, in our two-site cluster approximation (TCA) the Fourier-transform of the pair correlation function is obtained in the form

$$
\widehat{M}^{(2)}(q)=\widehat{F}^{(2)}\left(1-\hat{f}^{2}\right)^{-1}\left[1+(z-1) \hat{f}^{2}-\pi(q) \hat{f}\right]
$$

where

$$
\begin{aligned}
& \pi(q)=\sum_{j \in \pi_{i}} \mathrm{e}^{\mathrm{i} q\left(\boldsymbol{R}_{j}-\boldsymbol{R}_{i}\right),}, \pi(0)=z, \quad \hat{f}=\left(\widehat{F}^{(2)}\right)^{-1} \widehat{F}^{(11)}, \\
& \widehat{M}_{i j}^{(2)}=\left(\begin{array}{cc}
\left\langle\widehat{S_{i} S_{j}}\right\rangle^{\mathrm{c}} & \left\langle\widehat{S_{i} X_{j}}\right\rangle^{\mathrm{c}} \\
\left\langle\widehat{X_{i} S_{j}}\right\rangle^{\mathrm{c}} & \left\langle\widehat{\left\langle X_{i} X_{j}\right.}\right\rangle^{\mathrm{c}}
\end{array}\right), \\
& \left(\widehat{F}^{(2)}\right)_{\alpha \beta}=\left(\begin{array}{cc}
\left\langle\widehat{S_{i} S_{j}}\right\rangle_{H_{i}}^{c} & \left\langle\widehat{S_{i} X_{j}}\right\rangle_{H_{i}}^{c} \\
\left\langle\widehat{X_{i} S_{j}}\right\rangle_{H_{i}}^{c} & \left\langle\widehat{X_{i} X_{j}}\right\rangle_{H_{i}}^{c}
\end{array}\right), \\
& \left(\widehat{F}^{(11)}\right)_{\alpha \beta}=\left(\begin{array}{cc}
\left\langle\widehat{S_{i} S_{j}}\right\rangle_{H_{i j}}^{c} & \left\langle\widehat{S_{i} X_{j}}\right\rangle_{H_{i j}}^{c} \\
\left\langle\widehat{X_{i} S_{j}}\right\rangle_{H_{i j}}^{\mathrm{c}} & \left\langle{\widehat{X_{i} X_{j}}}_{H_{H_{i j}}}^{c}\right.
\end{array}\right), \quad\left(\left\langle\widehat{\left\langle S_{i} S_{j}\right.}\right\rangle^{\mathrm{c}}\right)_{\alpha \beta}=\left\langle S_{i \alpha} S_{j \beta}\right\rangle .
\end{aligned}
$$

Taking $q=0$ in Eq. (12) one obtains the known result [4]. The exact formula for the one-dimensional system $(z=2)$ in zero external field [5] also follows from Eq. (12). The same technique allows us to calculate the higher correlation functions also.

Let us formulate our main results. We express thermodynamic potential and correlation functions of the model via intracluster potentials and correlation functions. In this way our theory partially accounts for fluctuations and structure of the lattice. As a result, the theory distinguishes between magnetic properties of quenched and annealed systems in contrast to mean field approximation (MFA). The other peculiarity shows both Curie temperature and the temperature of the alloy binodal segregation. Figures 1 and 2 show phase diagrams of TCA and MFA for two-sort system at different parameters of the IIamiltonian. Apart from a complicated form of the TCA phase diagrams with respect to those of MFA one can also remark the rule that great value of the quantity $\widetilde{K}=K_{11}+K_{22}-2 K_{12}$ enhances both segregation and magnetization, whereas great $\widetilde{V}=V_{11}+V_{22}-$ $2 V_{12}$ enhances segregation and therefore enhances magnetization, if $\widetilde{K}>0$, and suppresses magnetization, if $\widetilde{K}<0$.

\section{References}

[1] R. Kubo, J. Phys. Soc. Japan 17, 1100 (1962).

[2] R.R. Levitskii, S.I. Sorokov, R.O. Sokolovskii, Ferroelectrics 153, 147 (1994).

[3] R.R. Levitskii, S.I. Sorokov, R.O. Sokolovskii, J. Magn. Magn. Mater. 140-144, 271 (1995).

[4] V.G. Vaks, N.E. Zein, Zh. Exp. Teor. Fiz. 67, 1082 (1974).

[5] B.Ja. Balagurov, V.G. Vaks, R.O. Zajtsev, Fiz. Tverd. Tela 16, 2302 (1974).

[6] T. Kawasaki, Prog. Theor. Phys. 58, 1357 (1977). 